\title{
Voice Disorders and Posturography: Variables to Define the Success of Rehabilitative Treatment
}

\author{
Ernesto Bruno, Alessandro De Padova, Bianca Napolitano, Patrizia Marroni, Raffaella Batelli, Fabrizio Ottaviani, \\ and Marco Alessandrini, Rome, Italy
}

\begin{abstract}
Summary. Previous studies have investigated the relationship between muscular tension, body posture, and voice quality. The aim of this paper is to study the postural pattern during voice production in healthy subjects compared with patients affected by voice disorders and in the same patients before and after vocal treatment by means of static posturography. Classic posturographic variables and spectral frequency analysis of body sway have been measured. Posturographic values in patients before vocal treatment and controls were within normal ranges but not homogeneous. Body sway significantly decreased during voice production in patients after voice training. Spectral frequency analysis of body sway showed a significantly decreased body sway at middle frequencies on the anteroposterior $(y)$ plane during voice production after voice training. Our results would suggest that in patients affected by voice disorders rehabilitative treatment may cause an improvement of the body proprioceptive scheme and this improvement might be useful to evaluate the proper (ongoing) treatment.
\end{abstract}

Key Words: Posture-Voice disorders-Spectral frequency analysis-Posturography-Proprioception.

\section{INTRODUCTION}

Posture has been established as an important component of the vocal performance.

Because postural alignment is not an inherent trait, it is possible to acquire a proper one through training and strengthening several muscle groups to obtain a good balance between the activity of agonistic and antagonistic muscles. ${ }^{1}$

During speech production, the activity of abdomen and chest muscles seems to impair the postural control. In fact, most voice volume changes are caused by the active emptying of the rib cage, produced by the contraction of the triangularis sterni and abdominalis muscles needed to optimize the inspiratory movement of the diaphragm, which is rapidly contracted during voice production to refill the respiratory system between phrases. $^{2}$

In particular, during speech production in upright position, the lateral region of the abdomen (oblique abdominal muscles) is actively contracted, mainly during loud speech production and during speech produced at low lung volumes. ${ }^{3,4}$ Furthermore, the studies on prephonatory dynamics show that there is an active rib cage enlargement that occurs during prephonatory posturing, that is not a passive response to abdominal compression. ${ }^{5,6}$ Moreover, there is clinical evidence that the presence of hypertonicity of extrinsic laryngeal muscles, of posterior weight bearing, of forward positioning of the head (all factors resulting in altered body posture) are predictive of severe dysphonia. ${ }^{7}$

The active contraction of the above-mentioned muscles may cause the loss of postural balance or a less-effective postural strategy during voice production as a result of an alteration of the body proprioceptive scheme even in completely healthy

\footnotetext{
Accepted for publication June 13, 2007.

From the Department of Otolaryngology, University of Rome "Tor Vergata", Roma, Italy.

Address correspondence and reprint requests to Ernesto Bruno, Department of Otolaryngology, Policlinico “Tor Vergata," Viale Oxford, 81, 00133 Roma, Italy. E-mail: bruno. ernesto@libero.it

Journal of Voice, Vol. 23, No. 1, pp. 71-75

0892-1997/\$36.00

(c) 2009 The Voice Foundation

doi:10.1016/j.jvoice.2007.06.002
}

subjects, and in patients affected by dysfunctional voice disorders.

It is possible to assess objectively the postural performance by means of static posturography, which records the forces applied by the subject's feet on a supporting surface to keep the body's center of gravity within the body's ground projection.

In previous studies, we proposed the spectral frequency analysis of the body sway in posturography to define specific postural patterns, each of them expressing the predominance of a specific sensorial input. ${ }^{8,9}$

The aim of the present paper is to study the postural pattern during voice production both in healthy subjects and in patients affected by voice disorders by means of static posturography and to verify whether any difference in postural pattern exists between healthy subjects and patients and whether any variation of postural pattern occurs between the same patients, before and after an adequate voice training. In addition, we would like to evaluate if there is a predominant sensorial input which is involved in maintaining posture when a new postural pattern is determined by the voice training.

\section{MATERIALS AND METHODS}

Fifteen consecutive patients (all female, mean age $39.5 \pm 3.1$ years) affected by dysfunctional voice disorders and never treated with vocal training constituted the study group. Ten healthy volunteer subjects, age- and sex-matched (all female, mean age $41 \pm 4.2$ years) constituted the control group.

Each patient and control underwent an accurate anamnesis for voice disorders, an ear, nose, and throat examination, a fiberoptic laryngoscopy, a videolaryngostroboscopy, and a vocal spectrography to diagnose or to exclude a dysfunctional voice disorder. All patients reported dysphonia, chronic or episodic, lasting for at least 2 years, with increased severity of symptoms three or four times per year usually during cold seasons or after vocal abuse. All patients reported that the voice disorder was causing social or working limitations. The severity of the disorder was assessed for each patient by the Voice Handicap Index (VHI) ${ }^{10}$ Control subjects never complained of dysphonia. The 
ear, nose, and throat examination did not reveal significant pathologies either in controls or in dysphonic patients. The fiberoptic laryngoscopy revealed the presence of vocal nodules in eight of the 15 selected patients, the presence of muscle tension dysphonia in seven of the 15 selected patients, and normal vocal folds morphology and motility in control subjects. The videolaryngostroboscopy revealed a normal mucosal pattern in controls and specific alterations in the group of patients. Vocal spectrography (speech filing system (SFS)-win program) revealed absence of harmonicas on high frequencies, often one unconstant fundamental frequency, presence of pneumophonic incoordination, and one important aperiodic component between a harmonica and the other in all patients. Moreover, all patients showed a hard vocal attack and a rather elevated intensity.

All the selected patients underwent a vocal training consisting of exercises for pneumophonic coordination; respiratory dynamics and relaxation of general muscular tone, in particular at the sternocleidomastoid and scapulo-omeral level together with all the exercises of phono-articulation settled by the standard protocol. The vocal training was performed twice a week for a period lasting 8-16 consecutive weeks. A control spectrography was performed at the end of the treatment.

Each selected patient and each control underwent a complete physical examination and blood analysis to exclude any systemic disease; an orthopedic examination; and an otoneurological investigation, according to the following protocol: accurate anamnesis, tonal liminar audiometry, assessment of spontaneous and evoked nystagmus by electronystagmographic recordings, and computerized static posturography.

The exclusion criteria, for both patients and controls, included neurological, systemic (alcohol addiction, thyroid disease, and lumbar root disease), or psychiatric disorders; presence of postural hypotension; visual acuity below 10/10; coexistence of labyrinthine symptoms (vertigo or dizziness); or pathological signs at standard vestibular tests (such as spontaneous or evoked nystagmus, abnormal response to the caloric vestibular tests, and alteration of the vestibulospinal reflex).

Static posturography was performed using a normalized computerized static posturography platform (S.Ve.P. Amplaid). Patients and controls were instructed to maintain an upright position during the test, first with eyes open, then with eyes closed, thereafter with eyes closed during voice production. Testing subjects in the eyes closed condition eliminates optic afference and maximally enhances the contribution of proprioceptive afferents. Patients were invited to pronounce the vocal "e" at high intensity for the period of examination. The recording period was 20 seconds for each test. Patients were tested before and after a period of vocal training.

The following variables have been calculated from the statokinesigram: (1) trace length: the length of the trace made by the center of gravity; (2) trace surface: $90 \%$ of the surface area covered by the trace; (3) mean velocity of body sway: mean velocity of the center of gravity; (4) standard deviation of mean velocity, and (5) fast Fourier transformation on $x$ (lateral) and $y$ (anteroposterior) planes (FFTX, FFTY) of the body sway, that is, the frequency domain analysis of body sway.
Graphic evaluation of spectrograms (two mobile vectors: $x / y$ ) was carried out using Autocad 13 software (Windows/Microsoft). With this procedure, the frequency of body oscillations (power spectra), ranging from 0.01 to $5.00 \mathrm{~Hz},{ }^{11}$ can be quantified.

The frequency spectrum was subdivided into three groups: $0.01-0.70,0.70-1.00$, and $1.00-5.00 \mathrm{~Hz}$. Within each group, the spectral intensity was determined by adding the relative power spectra and the mean $( \pm \mathrm{SD})$ power spectra obtained for each group.

All results are expressed as mean \pm SD. Posturographic values, including power spectra, were compared in patients before treatment and in controls and afterward in the same patient before and after treatment. Statistical analysis of mean differences was performed using unpaired $t$ test, while comparing patients and control and paired $t$ test while comparing patients before and after treatment (Excel-Windows), with a $p$ value of 0.01 or less defined as significant.

\section{RESULTS}

All patients showed an improved voice quality after treatment; vocal spectrography showed improved values in all the patients after treatment. The VHI score decreased in all patients (Table 1).

Posturography revealed a similar postural pattern in controls and patients before treatment. Furthermore, posturography revealed significantly increased body sway during voice production compared with the closed eyes condition in both controls and patients before treatment. A significantly decreased body sway, during voice production compared with the closed eyes condition, was instead observed in many patients after voice training.

\section{Posturographic variables $(a, b, c$, and $d)$}

Patients before training and controls showed a significant increase of trace length, trace surface, and mean velocity during voice production when compared with the closed eyes condition. Patients after vocal treatment showed a significant decrease of trace length and mean velocity during voice production when compared with the closed eyes condition and with the same patients during voice production before vocal treatment (Table 2, Figures 1-3).

The variable "trace surface" is less homogeneous than the above-mentioned variables in patients after treatment when compared with patients before treatment and during voice production. These variations will be discussed in the next section. In fact, trace surface showed increased values in six out of 15 subjects; decreased values in five out of 15 patients; and no significant variations were recorded in the last four patients.

\section{Frequency analysis (e)}

Patients after treatment showed significantly decreased body sway during voice production when compared with the closed eyes condition and when compared with patients performance before treatment but only on the anteroposterior $(y)$ plane whereas no significant variations were recorded on the lateral (x) plane. 
TABLE 1.

Patients Population Profile

\begin{tabular}{|c|c|c|c|}
\hline Diagnosis & $\begin{array}{c}\text { VHI Before } \\
\text { Therapy }\end{array}$ & $\begin{array}{l}\text { Weeks of } \\
\text { Treatment }\end{array}$ & $\begin{array}{l}\text { VHI After } \\
\text { Therapy }\end{array}$ \\
\hline 1. Vocal fold nodules & 52 & 16 & 35 \\
\hline 2. Vocal fold nodules & 34 & 8 & 18 \\
\hline $\begin{array}{l}\text { 3. Muscle tension } \\
\text { dysphonia }\end{array}$ & 48 & 16 & 31 \\
\hline 4. Vocal fold nodules & 39 & 8 & 23 \\
\hline $\begin{array}{l}\text { 5. Muscle tension } \\
\text { dysphonia }\end{array}$ & 62 & 16 & 40 \\
\hline $\begin{array}{l}\text { 6. Muscle tension } \\
\text { dysphonia }\end{array}$ & 50 & 16 & 32 \\
\hline $\begin{array}{l}\text { 7. Muscle tension } \\
\text { dysphonia }\end{array}$ & 47 & 16 & 34 \\
\hline $\begin{array}{l}\text { 8. Muscle tension } \\
\text { dysphonia }\end{array}$ & 44 & 16 & 30 \\
\hline 9. Vocal fold nodules & 36 & 16 & 27 \\
\hline $\begin{array}{l}\text { 10. Muscle tension } \\
\text { dysphonia }\end{array}$ & 48 & 16 & 32 \\
\hline 11. Vocal fold nodules & 35 & 16 & 25 \\
\hline $\begin{array}{l}\text { 12. Muscle tension } \\
\text { dysphonia }\end{array}$ & 45 & 16 & 36 \\
\hline 13. Vocal fold nodules & 49 & 8 & 36 \\
\hline 14. Vocal fold nodules & 50 & 8 & 34 \\
\hline 15. Vocal fold nodules & 50 & 8 & 38 \\
\hline
\end{tabular}

The decreased body sway was observed at low and middle frequencies, with a higher significance for the middle ones ( $P=0.24$ for low frequencies; $P<0.01$ for middle frequencies $)$ (Table 3, Figure 4).

\section{DISCUSSION}

Recent studies have showed a closed relationship between posture and voice production.

Muscular activity during voice production may cause an impaired postural performance, because of altered coordination

TABLE 2.

Mean \pm SD of Different Posturographic Variables (Trace Length, Trace Surface, and Mean Velocity) in Patients Before and After Treatment, in Different Examination Conditions

\begin{tabular}{|c|c|c|c|}
\hline & Trace Length & Trace Surface & $\begin{array}{l}\text { Mean } \\
\text { Velocity }\end{array}$ \\
\hline \multicolumn{4}{|c|}{ Before treatment } \\
\hline OE & $248.64 \pm 32.54$ & $156.63 \pm 43.38$ & $9.79 \pm 2.43$ \\
\hline CE & $336.18 \pm 43.49$ & $244.33 \pm 45.15$ & $13.23 \pm 3.04$ \\
\hline CE + voice & $413.35 \pm 31.55$ & $273.88 \pm 55.65$ & $16.01 \pm 1.85$ \\
\hline \multicolumn{4}{|c|}{ After treatment } \\
\hline $\mathrm{OE}$ & $264.53 \pm 27.44$ & $153.27 \pm 35.26$ & $10.14 \pm 2.27$ \\
\hline CE & $440.57 \pm 39.06$ & $240.11 \pm 41.5$ & $17.34 \pm 2.91$ \\
\hline CE + voice & $330.72 \pm 29.55$ & $227.14 \pm 58.77$ & $13.02 \pm 1.67$ \\
\hline
\end{tabular}

Abbreviations: $\mathrm{OE}$, open eyes; $\mathrm{CE}$, closed eyes; $\mathrm{CE}+$ voice, closed eyes while voice producing.

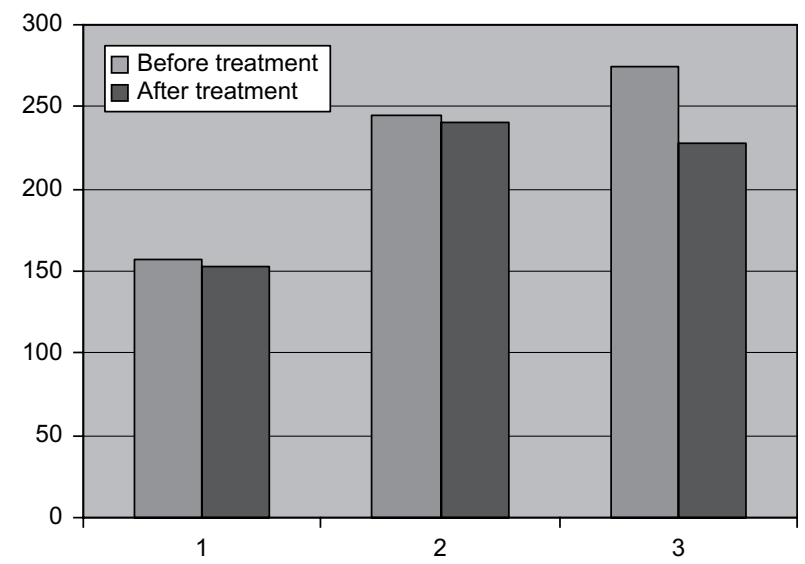

FIGURE 1. Mean of trace surface in selected patients, before and after voice treatment in different examination conditions $(1-$ open eyes, 2 -closed eyes, 3 -closed eyes while voice producing).

between breathing and phonation leading to an altered proprioceptive scheme of the body.

Correct voice training includes breathing exercises, because a correct breathing behavior is the basis for voice production: patients must learn to correctly refill respiratory system before talking and before the lung volume becomes too low to have a good phonation. They must learn to relax hypertonic muscles, because this can lead to posterior weight bearing and forward positioning of the head. Therefore, voice training implies that more attention is paid to the postural scheme of the body.

Analysis of posture by static posturography provides information on overall postural performance with variables such as trace length, surface, and velocity of body sway. It has been suggested that the labyrinth is a main monitor of the surface of body sway, whereas proprioception is a principle monitor of the velocity of body sway. ${ }^{12,13}$

In the present investigation, we analyzed the postural strategy in healthy subjects and in patients affected by dysfunctional voice disorders which were never treated with vocal training. The posturographic analysis showed no substantial differences in the two groups. Then we hypothesized that postural pattern

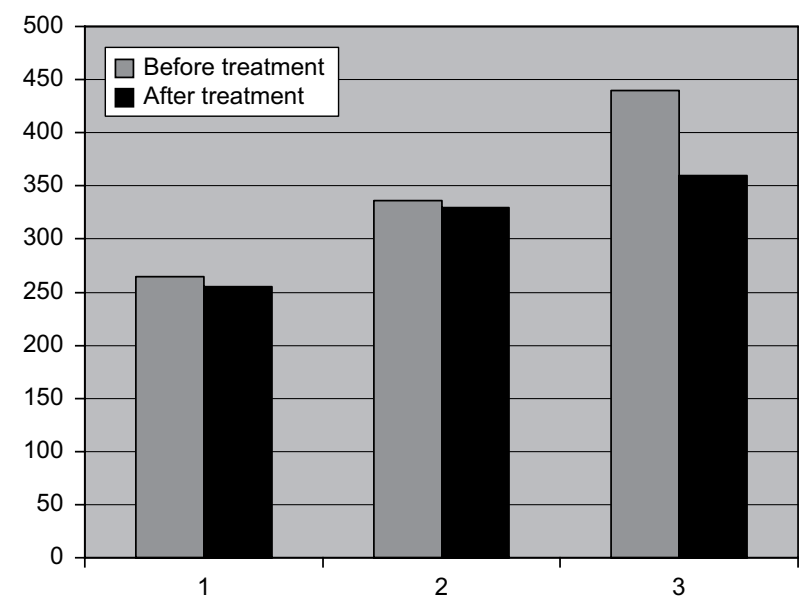

FIGURE 2. Mean of trace length in selected patients, before and after voice treatment in different examination conditions (1-open eyes, 2 -closed eyes, 3 -closed eyes while voice producing). 


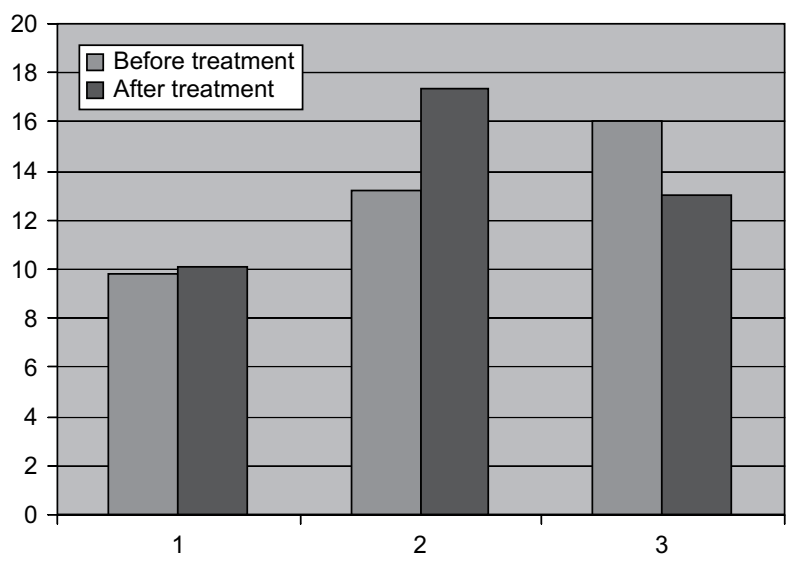

FIGURE 3. Mean velocity in selected patients, before and after voice treatment in different examination conditions $(1-$ open eyes, 2 -closed eyes, 3 - closed eyes while voice producing).

may exhibit variations in patients affected by voice disorders when receiving voice treatment and we demonstrated an improvement of postural performance in these patients after treatment. The improvement of postural strategy, showed by the decrease of posturographic values such as trace length and mean velocity after voice training, suggests an increased attention of these patients in postural rearrangement. This condition might be explained as the effect of the changed and improved control on respiration, diaphragmatic movements, and voice production by these patients.

Because proprioception is considered a principle monitor of the velocity of body sway, the decreased mean velocity of body sway observed in patients after vocal treatment and during voice production suggests an improvement of proprioceptive scheme of the body likely due to this treatment.

The trace surface was decreased in five out of 15 patients: these five patients performed a voice production without the need of breathing during the test. Moreover, these five patients received longer voice training than the others (16 continued weeks vs 8 weeks) and reported an overall better vocal performances after rehabilitation. The six subjects showing an increased trace surface needed to breathe during the test while performing a voice production. In these six patients, wider oscillations of the center of gravity have been recorded, in particular related to respiratory acts and likely due to the quick and strong contraction of diaphragm and abdominal muscles during inspirations needed to refill the respiratory system. The discrepancy of data may be explained by considering the fact that posturographic system measures the $90 \%$ of the surface area covered by the trace, but excluding the wider oscillations associated with respiratory acts and graphically measuring the surface of statokinesigrams between the respiratory acts; the trace surface results decreased during voice production in accordance with the first group considered.

In conclusion, these data seem to suggest that a decreased surface is a predictive index of a successful rehabilitative treatment. Moreover, previous studies showed that labyrinth is a main monitor of the surface of body sway, whereas in our selected patients it has been hypothesized that proprioception is the main sensorial input involved in the new postural strategy: therefore, the discrepancy of data may be the expression of the various subsystems involved in maintaining posture.

Frequencies of body sway are analyzed and quantified by FFT. ${ }^{14}$ Spectral frequency analysis of body sway seems to identify ranges of low, middle, and high frequencies which are thought to be influenced in varying degrees by different sensory inputs.

Low frequencies are mainly under vestibular control, whereas higher frequencies are guided by proprioceptive information. ${ }^{11}$ Proprioception seems to control mainly the anteroposterior sway when vestibular inputs are maintained. ${ }^{12}$ In our patients, spectral frequency analysis of body sway showed a significantly decreased body sway at middle frequencies on the anteroposterior $(y)$ plane during voice production after voice training. As proprioceptive control seems to manifest mainly on middle frequencies and on sagittal plane,${ }^{8}$ the present results would suggest that in patients affected by dysfunctional voice disorders, the voice rehabilitative treatment may cause an improvement of the proprioceptive scheme of the body.

In conclusion, in patients affected by dysfunctional voice disorders, posture is grossly maintained but a decrease of posturographic values such as trace length and mean velocity after voice training suggests a less effort needed to achieve this goal, likely due to the increased attention of these patients in postural rearrangement. In addition, spectral frequency analysis confirms that a more effective postural strategy after voice rehabilitative treatment is obtained improving the proprioceptive sensorial input.

Because the proprioceptive scheme can be altered by the muscular contraction during voice production, we think that

TABLE 3.

Mean \pm SD of the Power Spectra on $x$ and $y$ Plane at Low and Middle Frequencies in Patients After Treatment in Different Examination Conditions

\begin{tabular}{lrrrr} 
& \multicolumn{2}{c}{ Low Frequency } & \multicolumn{2}{c}{ Middle Frequency } \\
\cline { 2 - 5 } & $x$ & $y$ & $x$ & $y$ \\
\hline CE & $22.202 \pm 1.01$ & $21.984 \pm 0.87$ & $8.792 \pm 084$ & $8.676 \pm 0.69$ \\
CE + voice & $20.55 \pm 0.95$ & $18.5 \pm 0.9$ & $10.098 \pm 0.71$ & $4.406 \pm 0.42$ \\
\hline
\end{tabular}

Abbreviations: $\mathrm{CE}$, closed eyes; $\mathrm{CE}+$ voice, closed eyes while voice producing. 


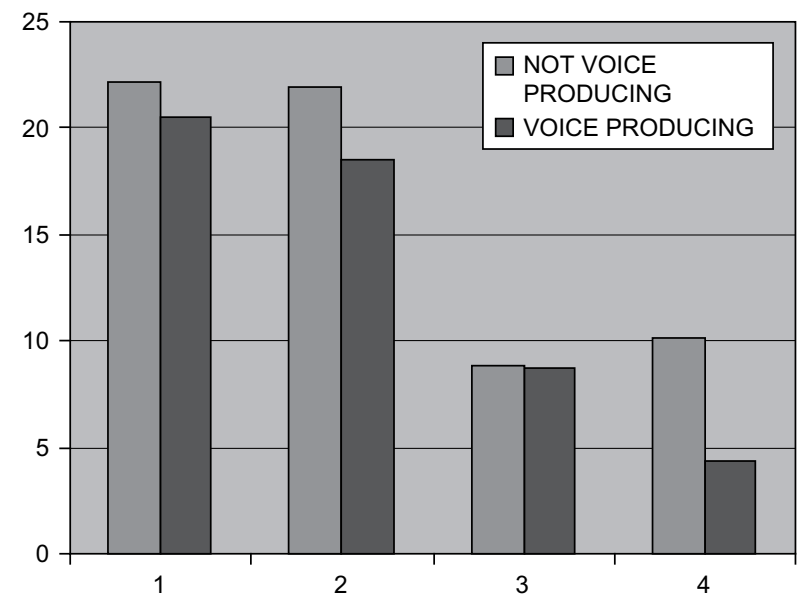

FIGURE 4. Frequency distribution of body sway in patients after treatment, in different examination conditions. 1-Mean of the power spectra on $x$ plane at low frequency range $(0.01-0.7 \mathrm{~Hz}) ; 2-$ Mean of the power spectra on $y$ plane at low frequency range $(0.01-0.7 \mathrm{~Hz})$; 3 - Mean of the power spectra on $x$ plane at middle frequency range $(0.7-1.0 \mathrm{~Hz})$; 4 - Mean of the power spectra on $y$ plane at middle frequency range $(0.7-1.0 \mathrm{~Hz})$.

voice therapy itself, through teaching a correct breathing behavior, through the relaxation of hypertonic muscles both before and during voice production, and through an improvement of muscular contraction self-consciousness, can improve patients' postural patterns while producing voice.

We believe that in clinical practice, the analysis of the variations of the posturographic pattern of patients before, during, and after voice training can be a useful variable to be considered by voice therapists to evaluate the proper ongoing of the treatment.

\section{REFERENCES}

1. Schneider CM, Dennehy CA, Saxon KG. Exercise physiology principles applied to vocal performance: the improvement of postural alignment. J Voice. 1997;11:332-337.

2. Estenne M, Zocchi L, Ward M, Macklem PT. Chest wall motion and expiratory muscle use during phonation in normal humans. J Appl Physiol. 1990;68:2075-2082.

3. Hoit JD, Plassman BL, Lansing RW, Hixon TJ. Abdominal muscle activity during speech production. J Appl Physiol. 1988;65:2656-2664.

4. Hoit JD. Influence of body position on breathing and its implications for the evaluation and treatment of speech and voice disorders. $J$ Voice. 1995;9: 341-347.

5. Cavallo SA, Baken RJ. Prephonatory laryngeal and chest wall dynamics. J Speech Hear Res. 1985;28:79-87.

6. Baken RJ, Cavallo SA, Weissman KL. Chest wall movements prior to phonation. J Speech Hear Res. 1979;22:862-871.

7. Kooijman PG, de Jong FI, Oudes MJ, Huinck W, van Acht H, Graamans K. Muscular tension and body posture in relation to voice handicap and voice quality in teachers with persistent voice complaints. Folia Phoniatr Logop. 2005;57:134-147.

8. Alessandrini M, Giacomini P, Sorace F, Bruno E. Postural stabilization after otolithic repositioning for posterior semicircular canal canalization. Acta Otorhinolaryngol Ital. 1998;18:368-372.

9. Alessandrini M, D’Erme G, Bruno E, Napolitano B, Magrini A. Vestibular compensation: analysis of postural re-arrangement as a control index for unilateral vestibular deficit. Neuroreport. 2003;14:1075-1079.

10. Jacobson BH, Johnson A, Grywalsky C, Silbergleit A, Jacobson G, Benninger MS. The Voice Handicap Index (VHI): development and validation. Am J Speech Lang Pathol. 1997;6:66-70.

11. Kohen-Raz R, Himmelfarb M, Tzur S, Shub Y. An initial evaluation of fatigue and circadian changes as assessed by multiple posturography. Percept Mot Skills. 1996;82:547-577.

12. Nagakawa H, Ohashi N, Watanabe Y, Mizukoshi K. The contribution of proprioception to postural control in normal subjects. Acta Otolaryngol (Stockh) 1993;(suppl 504):112-116.

13. Yasuda T, Nagakawa T, Inoue $\mathrm{H}$, Iwamoto $\mathrm{M}$, Inokuchi $\mathrm{A}$. The role of the labyrinth, proprioception and plantar mechanosensor in the maintenance of an upright posture. Eur Arch Otorhinolaryngol. 1999;256:527-532.

14. Yoneda S, Tokomasu K. Frequency analysis of body sway in the upright posture. Acta Otolaryngol. 1986;102:87-92. 